\title{
Modeling of intracranial vessels and Simulation of cerebral blood flow
}

\author{
Yingying Yan, Li Ke*, Qiang Du, Xiaodi Ding, Jia Chen \\ School of Electrical Engineering, Shenyang University of Technology, Shenyang, 110870, China
}

\begin{abstract}
The stable regulation of cerebral blood flow plays an important role in the normal operation of brain function. The disturbance of cerebral blood flow automatic regulation will lead to brain injury and lead to cerebrovascular disease. Therefore, it is of practical clinical significance to study the fine modeling of intracranial blood vessels. First of all, based on the anatomic structure of the intracranial blood vessels, the above sagittal sinus vein, sigmoid sinus, superior petrosal sinus, transverse sinus and cerebral arterial circle were mainly modeled, the three-dimensional model of cerebral blood flow is constructed. Secondly, the three-dimensional model is given conductivity characteristics. Through the expansion and contraction of cerebral blood vessels to simulate the self-regulation of cerebral blood flow, the simulation method of cerebral blood flow impedance is studied. When the blood flow changes, the brain impedance is calculated. The simulation data shows that the change trend of the electric potential and the whole brain impedance of the outer layer of the brain is consistent with the theoretical analysis. The experimental results show that the impedance curves and changes calculated by the brain model in this study are consistent with the measured impedance results, which shows that the modeling method in this paper is precise and effective, and provides a theoretical basis for further study of cerebral blood flow problems.
\end{abstract}

\section{Introduction}

Brain disease has great harm to human health. The most common brain disease in clinic is cerebrovascular disease. Cerebrovascular disease is a common and frequently occurring disease that endangers human life and health. It has the characteristics of high morbidity, high disability rate, high mortality rate and high recurrence rate. It is also a major disease of disability and death in middle-aged and elderly people ${ }^{[1]}$.

The occurrence of cerebrovascular disease is directly related to abnormal cerebral blood flow parameters and dysregulation of cerebral blood flow autonomy, the criterion for measuring the blood supply to the brain is cerebral blood flow. Once the cerebral blood flow decreases, the oxygen supply function of brain cells will also decrease, which will cause the functional changes of the brain ${ }^{[2]}$. If some part of the intracranial blood vessels become occluded by lesions themselves, it will lead to complete blockage of this part of blood flow in a shorter time, and local brain tissue necrosis will occur, long-term intracranial blood supply or abnormal blockage of cerebral blood flow have serious consequences on human life and health and even lead to death ${ }^{[3]}$. Therefore, it is of great significance to study the fine modeling of intracranial vessels and the simulation method of cerebral blood flow impedance.

The construction of the cerebral blood flow simulation model mainly involves the electromagnetic and geometric characteristics, so the structure of the cerebral blood flow simulation model must be close to the real physiological structure and electrical characteristics of the brain and intracranial blood vessels. Meanwhile, in order to improve the calculation speed, the model construction should simplify the craniocerebral partition structure and the capillary position as much as possible. In the present study, simulating the human head from the earliest simple spherical models ${ }^{[4]}$ to multilayer concentric sphere models, and real brain models in recent years ${ }^{[5]}$, it can not only calculate the electromagnetic characteristics of the brain, but also be used to monitor brain injury ${ }^{[6]}$. In recent years, Hawthorne Christopher ${ }^{[7]}$ et al. and the research group of Institute of Instrumental Science and Electrical Engineering of Jilin University ${ }^{[8]}$ have studied the cerebral blood flow from the aspects of intracranial pressure bioimpedance measurement and noninvasive measurement of cerebral blood flow, respectively. The existing models mainly focus on the division of brain parenchyma for intracranial construction, and study the influence of conductivity of brain, cerebellum and cerebrospinal fluid on impedance. This study aims at the in-depth study of intracranial vascular construction and its own characteristic changes, which is more detailed and accurate than the original model analysis of cerebral blood flow. the fine structure of intracranial vessels is constructed for simulation calculation and research.

In this paper, the real structure of human skull and the anatomical structure of intracranial veins are taken as

\footnotetext{
$\overline{{ }^{*} \text { Corresponding author: keli@sut.edu.cn }}$
} 
prototypes, through the splicing of multiple ellipses, spheres, circles and cylinders, based on the structures of the sagittal sinus vein and transverse sinus vein, a 4-layer three-dimensional craniocerebral model was constructed, which approximated the true intracranial vascular structure and had the outline of the craniocerebral outer layer. By contracting and expanding the blood vessel volume, the function of autonomous regulation of cerebral blood flow is simulated to provide a theoretical model for the simulation of cerebral blood flow impedance.

\section{Construction of brain model}

\subsection{Intracranial vascular structure}

There are many curved fissures on the surface of the cerebral hemisphere called sulcus, and the protruding part of them is called gyrus ${ }^{[9]}$. Brain blood vessels attach to the sulcus and gyrus. Brain blood vessels are formed by an interlaced network of arteries and veins. The arteries of the brain arise from the internal carotid and vertebral arteries ${ }^{[10]}$, both form a cerebral arterial circle at the base of the brain. The direction of cerebral veins is different from that of arteries, with thin walls and no valves. The return of cerebral venous blood, mainly to the dural sinus, then to the heart via the internal jugular vein. In this paper, the change of intracranial blood flow is reflected by changing the radius of intracranial blood vessels, so as to obtain the overall cerebral blood flow. Due to the extremely curved shape of the arteries and veins entering the cranial cavity, the difficulty of comprehensive transformation and the speed of calculation, the vessels with more regular shape and less curvature were mainly selected as the study objects.

When modeling blood vessels, not only physiological structural characteristics but also physiological characteristics $^{[11]}$ need to be taken into account. For example, superior sagittal sinus vein embolism is the most common, often caused by dehydration and head trauma in children. When transverse sinus embolism occurs $^{[12]}$, cerebral venous reflux disorder may occur, causing severe cerebral congestion. Another example is the sigmoid sinus, when the scalp is infected, it is very likely to cause abnormal sigmoid sinus, thus causing abnormal brain function ${ }^{[13]}$. Cerebral arterial circle is also important for the analysis of deep blood supply and intracranial hemorrhage ${ }^{[14]}$. Therefore, in the selection of veins for modeling and drawing, veins with less curvature and more regular shape are selected as the main veins. It is composed of superior sagittal sinus vein, transverse sinus, superior petrosal sinus and sigmoid sinus. In the selection of arteries, cerebral arterial circle in the deep part were selected to form the vascular model, as shown in Fig 1.

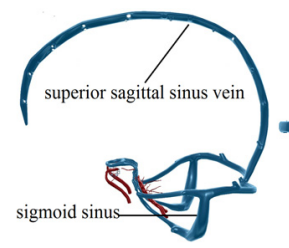

(a)

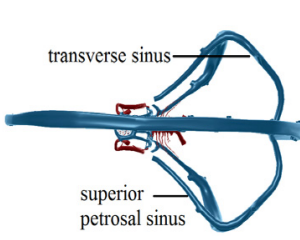

(b)

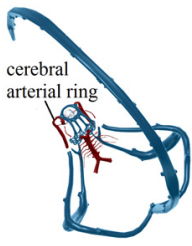

(c)
Fig.1 Anatomy of intracranial vessels

(a) Superior sagittal sinus vein and sigmoid sinus

(b) Transverse sinus and superior petrosal sinus

(c) Cerebral arterial circle

\subsection{Intracranial vascular construction}

Construction of intracranial vessels: transverse sinus, superior petrosal sinus, and sigmoid sinus. The sigmoid sinus lies between the two layers of dura mater in the sigmoid sulcus of the mastoid part of the temporal bone, as shown in Fig. 2(a). Draw a cylindrical body with a radius of $2.75 \mathrm{~mm}$ and a height of $5 \mathrm{~cm}$ at the drawing interface $(3,3.9,-7.2)$, rotate and mirror changes, and draw the left and right sigmoid sinus. The transverse sinus is a large dural venous sinus located between two layers of dura mater at the attachment edge of the tentorium cerebellum and contained in the transverse sinus sulcus on the inner surface of the skull. Starting from the occipital carina, the right transverse sinus usually continues in the superior sagittal sinus, while the left transverse sinus continues in the straight sinus. Draw a circle with an outer ring radius of $6.5 \mathrm{~cm}$ and an inner ring radius of $2.75 \mathrm{~mm}$ at $(1.2,0,-1)$. The transverse sinus and superior petrosal sinus veins are obtained by mirror and difference sets, as shown in Fig. 2(b).

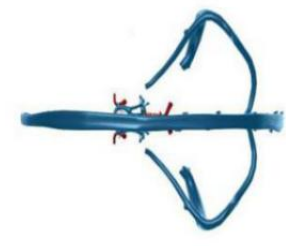

(a)

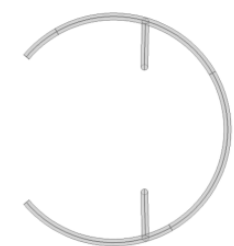

(b)
Fig. 2 Construction of intracranial vessels

(a) Top view of cerebral vascular anatomy

(b) Construction of transverse sinus, superior petrosal sinus and sigmoid sinus

The superior sagittal sinus vein was constructed, and the superior sagittal sinus was located at the attachment of the flange of the falx cerebri, as shown in Fig. 3(a). Draw a ring with an outer ring radius of $6.5 \mathrm{~cm}$ and an inner ring radius of $2.75 \mathrm{~mm}$ at the drawing interface $(1.5$, $0,-1)$, and a ring with an outer ring radius of $12 \mathrm{~cm}$ and an inner ring radius of $2.75 \mathrm{~mm}$ at the position $(1.5,0$, -4.5). The two rings were matched with the position and angle of the superior sagittal sinus vein by rotation, mirror and boolean operation, and the results after construction were shown in Fig. 3(b). 


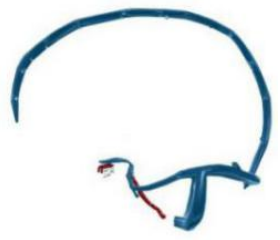

(a)

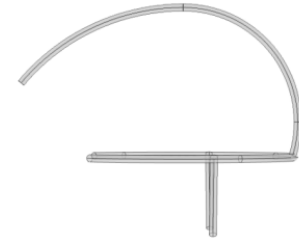

(b)
Fig.3 Construction of intracranial vessels (a) Anatomical side view of cerebral vessels (b)Construction of superior sagittal sinus vein

To construct deep intracranial vascular network, cerebral artery circle, located below the base of the brain, above the sella, around the gray nodule of the optic chiasm and the papillary body, as shown in Fig. 4(a). In the drawing interface $(-3.7,1.3,-1),(-3.6,1.3,0.25)$, $(-2.2,0,1.15)$ draw a cylinder with a bottom radius of $2.75 \mathrm{~mm}$ and a height of $3 \mathrm{~cm}$ at the position, with an outer ring radius of $1 \mathrm{~cm}$, an inner ring radius of $2.5 \mathrm{~mm}$, an outer ring radius of 180 degrees and a ring with an outer ring radius of $2 \mathrm{~cm}$, an inner ring radius of $2.5 \mathrm{~mm}$ and a radian of 140 degrees. The fitting structure of deep cerebral arterial circle is obtained by mirror transformation transformation. The results of the constructed intracranial vessels are shown in Fig.4(b).

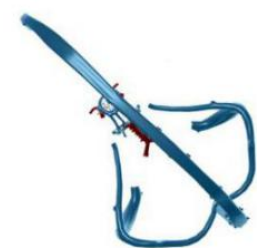

(a)

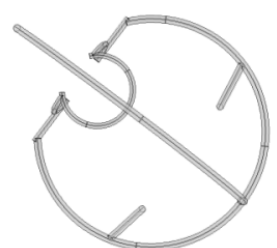

(b)
Fig. 4 Construction of intracranial vessels

(a) Anatomical maps of deep cerebral vessels

(b) Result map of intracranial vascular construction

\subsection{Construction of skull layer, scalp layer and brain parenchyma}

The skull is the skeleton of the human head, forming the overall contour of the brain, while the skin is a soft organ attached to the skull, white matter, gray matter, cerebrospinal fluid and intracranial blood vessels are filled in the skull. So in order to simulate and simulate the cerebral blood flow analysis model which is close to the real geometry, the first step is to analyze the composition and structural characteristics of the skull ${ }^{[15]}$, and construct the skull model. The models were modified by occipital bone, craniofacial bone and eye socket respectively ${ }^{[16]}$, and finally the skull structure with eye socket structure and craniofacial bone was obtained.

The cerebral cortex is the highest-level center that regulates or controls somatic movement. The cerebral cortex plays an important role in thermoregulation ${ }^{[17]}$. Body temperature can be regulated by conditioning. Once the cerebral cortex is lost, its normal life activities cannot be maintained. Considering its effect on impedance, the scalp is essential in the process of model building. The craniocerebral model was scaled so that the thickness of the scalp was about $0.5 \mathrm{~cm}$.
In this work, intracranial blood vessels are mainly analyzed to study cerebral blood flow. Therefore, in this study, the brain parenchyma is considered as a whole, and the skull layer is transformed to get its thickness of 1 $\mathrm{cm}^{[18]}$, and the constructed model is shown in Fig. 5.

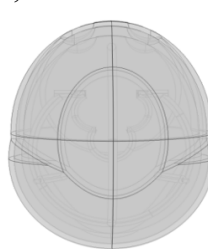

(a)

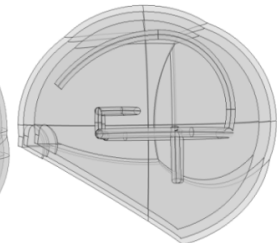

(b)

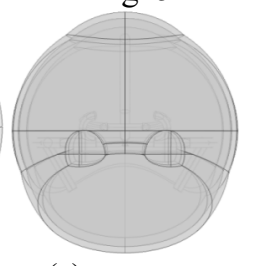

(c)
Fig.5 Head model

(a) Top view, (b)Left view, (c)Front view

\section{Analysis of craniocerebral impedance and blood flow changes}

\subsection{Electrical properties of brain tissue}

In this paper, the craniocerebral impedance detection is to measure the scalp potential caused by the excitation of a safe current in the human head ${ }^{[19]}$. Using the obtained potential value and the human brain simulation model, the craniocerebral impedance changes and the relationship with blood flow are calculated.

During the measurement of craniocerebral impedance, the frequency of the excitation current can be selected between $1-190 \mathrm{kHz}^{[20]}$, and the amplitude of the output excitation current can be set between $0-1.25 \mathrm{~mA}$ to meet the safety requirements of anthropometry. The system also detects the amplitude information of the boundary voltage. In clinical practice, $50 \mathrm{kHz}$ frequency excitation sinusoidal current signal is commonly used as the input signal to obtain scalp potential.

The dielectric constant and conductivity of each layer of the brain are significantly different due to its different tissue components ${ }^{[21]}$. The electrical properties of human brain and intracranial tissues under $50 \mathrm{kHz}$ excitation frequency are shown in Tab.1. The main structures of the cerebral blood flow analysis model include the scalp, skull, brain parenchyma, and main intracranial vessels.

Tab.1 Dielectric Properties of Human Brain and Major Intracranial Tissues at $50 \mathrm{kHz}^{[22]}$

\begin{tabular}{|c|c|c|}
\hline $\begin{array}{c}\text { Dielectric } \\
\text { layer }\end{array}$ & $\begin{array}{c}\text { Conductivity } \\
(\mathrm{S} / \mathrm{M})\end{array}$ & $\begin{array}{c}\text { Relative dielectric } \\
\text { constant (F/M) }\end{array}$ \\
\hline Atmosphere & 0 & 1 \\
\hline Scalp & 0.3 & 360 \\
\hline Skull & 0.02 & 150 \\
\hline $\begin{array}{c}\text { Brain } \\
\text { parenchyma }\end{array}$ & 0.2 & 80 \\
\hline Blood & 0.7 & \\
\hline
\end{tabular}

\subsection{Dynamic modeling of changes in craniocerebral impedance}

The change of craniocerebral impedance, as a function of time, is a combined effect of heart pumping, blood and vascular resistance ${ }^{[23]}$. Assuming that each contraction 
and diastole of the heart is a regular and constant process, ignoring the difference between each person, the periodic change of brain impedance is related to the periodic beating of the heart, then the single change of brain impedance with the heartbeat is caused by the expansion or contraction of the vascular radius of the brain itself, which is closely related to the staging of the cardiac ejection process. When the inflow of arterial blood is larger than the outflow, the volume of cerebral blood vessels expands, the impedance of brain decreases, and when the inflow of arterial blood is smaller than the outflow, the volume of cerebral blood vessels decreases, the impedance of brain increases, and the change process is shown in Tab. 2.

Tab. 2 Changes of Cardiac Cycle, Arterial Blood Flow, Brain Volume and Impedance

\begin{tabular}{|c|c|c|c|}
\hline Heart state & $\begin{array}{c}\text { Arterial } \\
\text { blood flow } \\
\text { status }\end{array}$ & $\begin{array}{c}\text { Brain } \\
\text { vascular } \\
\text { volume } \\
\text { changes }\end{array}$ & $\begin{array}{c}\text { Brain } \\
\text { impedance } \\
\text { changes }\end{array}$ \\
\hline $\begin{array}{c}\text { Isovolumic systole } \\
(0.04 \sim 0.06 \mathrm{~s})\end{array}$ & $\begin{array}{c}\text { Inflow }< \\
\text { Outflow }\end{array}$ & Reduce & Rise \\
\hline $\begin{array}{c}\text { Rapid ejection } \\
\text { period (0.11s) }\end{array}$ & $\begin{array}{c}\text { Inflow }> \\
\text { Outflow }\end{array}$ & Expand & Decrease \\
\hline $\begin{array}{c}\text { Slow ejection } \\
\text { period (0.14s })\end{array}$ & $\begin{array}{c}\text { Inflow }< \\
\text { Outflow }\end{array}$ & Reduce & Rise \\
\hline $\begin{array}{c}\text { Prediastolic } \\
(0.036 \mathrm{~s})\end{array}$ & $\begin{array}{c}\text { Inflow }< \\
\text { Outflow }\end{array}$ & Reduce & Rise \\
\hline $\begin{array}{c}\text { Isovolumetric } \\
\text { diastole (0.06s })\end{array}$ & $\begin{array}{c}\text { Inflow }< \\
\text { Outflow }\end{array}$ & Reduce & Rise \\
\hline $\begin{array}{c}\text { Rapid filling period } \\
(0.11 \mathrm{~s})\end{array}$ & $\begin{array}{c}\text { Inflow }< \\
\text { Outflow }\end{array}$ & Reduce & Rise \\
\hline $\begin{array}{c}\text { Slow filling period } \\
(0.20 \mathrm{~s})\end{array}$ & $\begin{array}{c}\text { Inflow }< \\
\text { Outflow }\end{array}$ & Reduce & Rise \\
\hline $\begin{array}{c}\text { Atrial contraction } \\
(0.11 \mathrm{~s})\end{array}$ & $\begin{array}{c}\text { Inflow }< \\
\text { Outflow }\end{array}$ & Reduce & Rise \\
\hline
\end{tabular}

\subsection{Modeling of intracranial blood flow changes}

Rhythmic contraction and diastole of the heart drive the blood. When the heart contracts, blood is injected into the aorta from the left ventricle. Blood is distributed throughout the body through the arteries. During diastole, blood flows back to the right atrium through the vena cava system. As shown in Table 3, when normal adults are quiet, the cardiac blood flow velocity in normal adults is $5 \mathrm{~L} / \mathrm{min}^{[24]}$, with a heart rate of $60-80$ beats $/ \mathrm{min}$ and an average of 70 beats/min, the number of milliliters of ejection per heart is about $71 \mathrm{ml}$.

Tab. 3 The law of heart blood circulation

\begin{tabular}{|c|c|c|c|}
\hline $\begin{array}{l}\text { Cardiac } \\
\text { ejection }\end{array}$ & Flow site & Proportion & $\begin{array}{l}\text { Cardiac } \\
\text { Return }\end{array}$ \\
\hline \multirow{7}{*}{$\begin{array}{c}5 \mathrm{~L} / \mathrm{min} \\
\text { Left } \\
\text { ventricle } \\
\text { to aorta }\end{array}$} & Brain & $15 \%$ & \multirow{7}{*}{$\begin{array}{c}5 \mathrm{~L} / \mathrm{min} \\
\text { Vena cava } \\
\text { to right } \\
\text { atrium }\end{array}$} \\
\hline & Coronary circulation & $5 \%$ & \\
\hline & Bronchial tube & $5 \%$ & \\
\hline & $\begin{array}{l}\text { Stomach, intestine, } \\
\text { spleen, pancreas, liver }\end{array}$ & $30 \%$ & \\
\hline & Kidney & $20 \%$ & \\
\hline & Skeletal muscle & $15 \%$ & \\
\hline & $\begin{array}{l}\text { Skin, bone and bone } \\
\text { marrow }\end{array}$ & $10 \%$ & \\
\hline
\end{tabular}

Blood supply to the brain comes from the internal carotid and vertebral arteries ${ }^{[25]}$, normal people in a quiet state, the blood flow per $100 \mathrm{~g}$ of brain tissue is $50-60$ $\mathrm{ml} / \mathrm{min}$, and the total blood volume of cerebral circulation is about $750 \mathrm{ml} / \mathrm{min}$, which is equivalent to $15 \%$ of the total ejection of the heart into the brain. Therefore, it is estimated that the blood intake into the brain is $10.65 \mathrm{ml}$ each time. Combined with the time change of cardiac cycle, the changes of craniocerebral vascular volume with cardiac cycle are shown in Tab. 4 .

Tab. 4 Changes of cardiac cycle, cerebrovascular radius and total volume

\begin{tabular}{|c|c|c|c|}
\hline $\begin{array}{c}\text { Cardiac } \\
\text { cycle }\end{array}$ & Vein $(\mathrm{mm})$ & Artery $(\mathrm{mm})$ & $\begin{array}{c}\text { Total volume } \\
(\mathrm{cm} 3)\end{array}$ \\
\hline Base volume & 2.75 & 2.5 & 19.872 \\
\hline Ejection $4 \mathrm{ml}$ & 3 & 2.75 & 23.712 \\
\hline Ejection $8 \mathrm{ml}$ & 3.25 & 3 & 27.892 \\
\hline Ejection $10 \mathrm{ml}$ & 3.4 & 3.15 & 30.564 \\
\hline Return $1 \mathrm{ml}$ & 3.3 & 3.05 & 28.769 \\
\hline Return $3 \mathrm{ml}$ & 3.15 & 2.9 & 26.181 \\
\hline Return $4 \mathrm{ml}$ & 3.1 & 2.85 & 25.344 \\
\hline Return $5 \mathrm{ml}$ & 3.05 & 2.8 & 24.521 \\
\hline Return $7 \mathrm{ml}$ & 2.9 & 2.65 & 22.136 \\
\hline Return $8 \mathrm{ml}$ & 2.85 & 2.6 & 21.368 \\
\hline
\end{tabular}

\section{Simulation and result analysis}

\subsection{Simulation results and analysis of a single model}

The simulation model constructed above is used for simulation experiment. The scalp potential of the craniocerebral model is measured by four-electrode method. The forehead is placed for excitation and detection, and the occipital part is grounded.The model consists of four layers, and the conductivity and relative dielectric constant of the scalp, skull, brain parenchyma and blood are set in the same structure as the real brain. The excitation electrode is set to a current source of $50 \mathrm{kHz}, 0.01 \mathrm{~A}$. The simulation results are shown in Fig. 6 . The potential changes are measured in both scalp and intracranial vessels, which proves that the purpose of measuring craniocerebral impedance can be achieved by this simulation, and lays a foundation for further analysis of intracranial vessels and brain impedance problems.

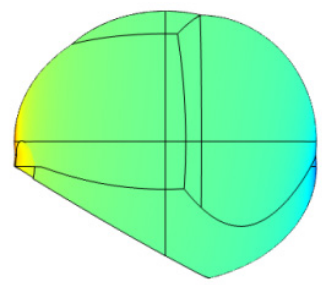

(a)

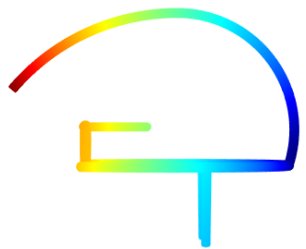

(b)
Fig.6 Brain simulation results.

(a) Scalp potential distribution map

(b) Intracranial vascular potential distribution map 


\subsection{Model simulation results and analysis of different vessel volumes}

After a simulation of the craniocerebral vascular model, the volume of the intracranial vessels was changed, all the set parameters were the same, and then the scalp potential changes were measured by applying the same electric field excitation. The results obtained by drawing the multiple measurement results according to the time are shown in Tab.5.

Tab.5 Scalp potential and calculated impedance corresponding to different intracranial vessel volume changes

\begin{tabular}{|c|c|c|}
\hline Volume $\left(\mathrm{cm}^{3}\right)$ & Scalp potential $(\mathrm{mv})$ & Impedance $(\Omega)$ \\
\hline 19.872 & 344.213 & 34.421 \\
\hline 23.712 & 344.048 & 34.405 \\
\hline 27.892 & 343.882 & 34.388 \\
\hline 30.564 & 343.751 & 34.375 \\
\hline 28.769 & 343.832 & 34.383 \\
\hline 26.181 & 343.928 & 34.392 \\
\hline 25.344 & 343.953 & 34.395 \\
\hline 24.521 & 343.990 & 34.399 \\
\hline 22.136 & 344.060 & 34.406 \\
\hline 21.368 & 344.112 & 34.411 \\
\hline
\end{tabular}

\subsection{Impedance Analysis of Brain Models with Different Volumes}

From the impedance values obtained in Tab.5, we can see that by changing the volume of intracranial vessels to simulate the cardiac cycle process, different models are applied with the same excitation, and finally corresponding impedance changes are obtained. When the heart contracts, the volume of intracranial blood vessels expands, and the overall impedance of the brain decreases. When the heart relaxes, the blood vessel volume decreases and the craniocerebral impedance increases. The results obtained after plotting the craniocerebral impedance data are shown in Fig.7.

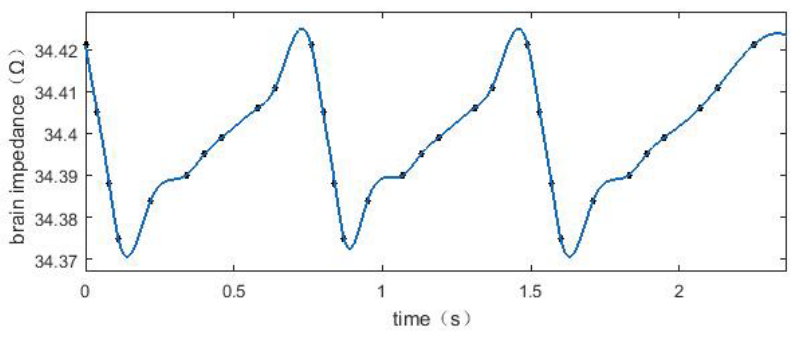

Fig. 7 Brain impedance simulation results

From Fig.7, it can be calculated that the heart rate is 79 beats/min and the impedance change value is 0.0463 . The calculated heart rate conforms to the normal heart rate range of $60-100$ beats $/ \mathrm{min}$. The impedance change values will be further verified experimentally.

\section{Detection experiment and result analysis}

\subsection{Data sources}

In this study, 10 healthy volunteers were recruited to participate in the experiment voluntarily and signed the informed consent before the experiment. The measuring principle and simulation model of brain impedance are consistent. The experiment verifies that the four-electrode method is used to measure brain impedance, electric field excitation is applied at the forehead, grounding at the occipital part, and the electrodes for detecting impedance are affixed to the inner side of the excitation and grounding end, respectively.

In this study, the measurement of brain impedance was performed by NICO100C module of BIOPAC. The acquisition environment needs to be quiet. The subjects were tested under closed-eye conditions, and other factors were excluded. The experimenters measured at different times, and each experimenter measured two groups of experiments. The measured impedance results are shown in Fig. 8(a). The measured impedance after deburring is shown in Fig. 8(b). The 20 groups of measurement data were analyzed for significance, and it was verified that the error met the normal distribution, and the data were statistically significant $(\mathrm{p}<0.01)$.

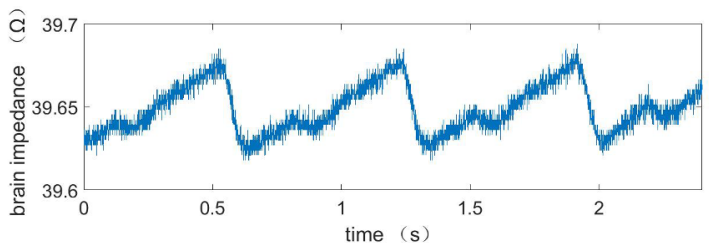

(a)

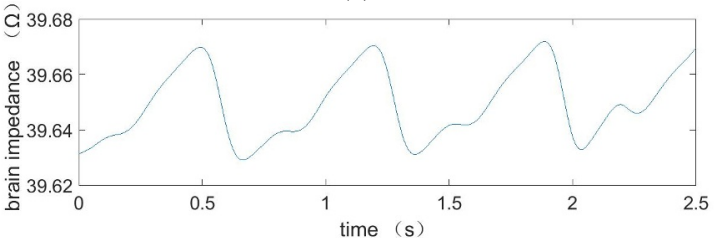

(b)

Fig. 8 Brain impedance measurement

(a) Measurement result

(b) Result chart after deburring

\subsection{Calculation of cerebral blood flow parameters}

20 sets of impedance measurement data are summarized and calculated, and the results are shown in Fig.9. It can be concluded that there are differences among different individuals, and that each person carries out tests at different measurement times, and the measurements will also be different.Among them, the difference rate of impedance change value was $0.43 \%$ to $32.8 \%$, the difference rate of heart rate was $1.23 \%$ to $15 \%$, and the difference rate of rapid ejection time was $0.75 \%$ to $18.05 \%$. 


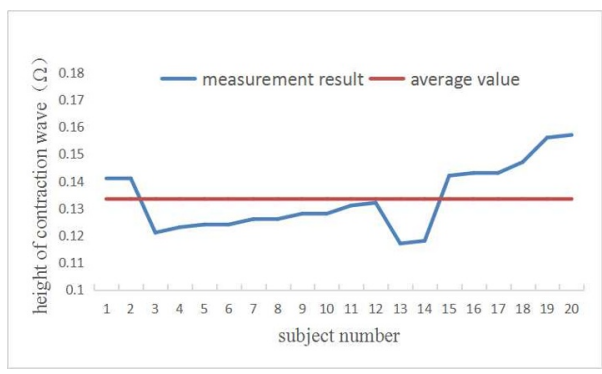

(a)

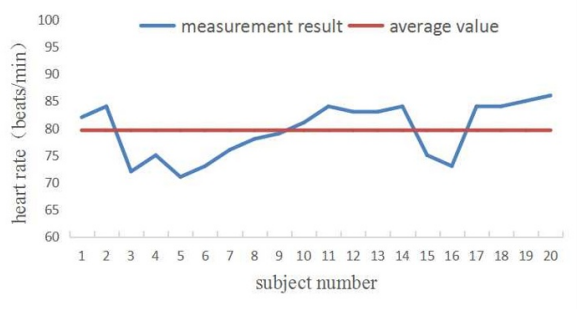

(b)

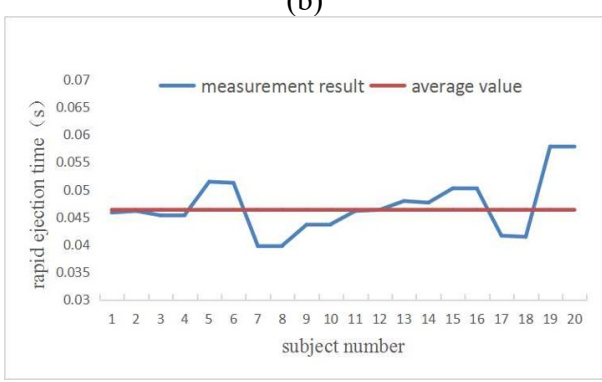

(c)

Fig. 9 Analysis results

(a) Impedance changes,(b) Heart rate, (c) Rapid ejection time

The average value of the measured data is compared with the experimental simulation results, and the results are shown in Tab.6. There was no significant difference between the measured impedance change value and the simulated impedance change value $(p>0.05)$, which was statistically significant. There was no significant difference between heart rate measurements and simulation results $(\mathrm{p}>0.05)$, and there was no significant difference between fast ejection time and simulation setting time $(p>0.05)$.

Tab. 6 Results of 20 groups of measured data

\begin{tabular}{|c|c|c|c|}
\hline & $\begin{array}{c}\text { Impedance } \\
\text { change value } \\
(\Omega)\end{array}$ & $\begin{array}{c}\text { Heart rate } \\
\text { (beats/min) }\end{array}$ & $\begin{array}{c}\text { Rapid ejection } \\
\text { time (s) }\end{array}$ \\
\hline $\begin{array}{c}\text { Measured } \\
\text { mean }\end{array}$ & 0.0537 & 80 & 0.133 \\
\hline $\begin{array}{c}\text { Simulation } \\
\text { result }\end{array}$ & 0.0463 & 79 & 0.115 \\
\hline $\begin{array}{c}\text { Error } \\
\text { Range }\end{array}$ & $0.19 \%-39.3 \%$ & $1.23 \%-15 \%$ & $0.75 \%-18.05 \%$ \\
\hline $\begin{array}{c}\text { Simulation } \\
\text { error }\end{array}$ & $13.78 \%$ & $4.81 \%$ & $13.53 \%$ \\
\hline
\end{tabular}

\section{Discussion}

In this paper, intracranial vascular modeling is the main simulation model, including three-dimensional and four-layer brain models of the scalp, skull, and brain parenchyma. Brain structure is very complex.Simple vascular modeling using MRA vessel images can not fully demonstrate the impedance characteristics of cerebral blood flow, although it shows the detailed characteristics of the vessel itself. Compared with pure vessel impedance study, the model in this study can also obtain the impedance information of the whole brain when obtaining the effective information of the brain blood vessels. The study of the autonomic regulation of cerebral blood flow is of great significance. Although the traditional brain model includes the division of skull and intracranial tissue, the study of cerebral blood flow impedance has certain limitations. The brain model in this paper can provide the impedance information caused by the changes of brain blood supply and blood flow.

Due to the expansion and contraction of intracranial blood vessel volume in the simulation process, the intracranial regions were not divided in the modeling of brain parenchymal layer. Many brain details have not been studied to improve computational speed and reduce computational error rates. During the simulation of cerebral blood flow changes, the process of cerebral blood supply is simulated by manually changing the volume of blood vessels. The simulated changes can not fully describe the process of cerebral blood supply, including the details of cardiac rehab, and the process of blood supply needs to be further studied.

As shown in Tab.5, when the intracranial blood flow changes by $1 \mathrm{ml}$, the impedance and the potential changes in millivolt level can be detected on the scalp.In Table 6, the simulation results are within the actual measurement error range, which further illustrates the effectiveness of the simulation results. The relationship between cerebral blood flow and cerebral impedance can be well established by craniocerebral modeling. The study of cerebral blood supply is an important part of brain health research. The model proposed in this paper provides a prerequisite for impedance detection of cerebral blood flow. Through the analysis of simulation and experimental results, more refined models can obtain more accurate results. Placing a dynamic vascular model inside the brain model can enable the brain model to provide continuous information of cerebral blood supply impedance. If the impedance of the patient's brain is continuously measured by this method, the change of intracranial blood flow can be analyzed, which has practical clinical significance.

\section{Conclusion}

Starting from the true cranial and intracranial vascular structure of human body, this study constructed a craniocerebral model with the sagittal sinus vein, superior petrosal sinus, sigmoid sinus, transverse sinus and cerebral arterial circle as the main body and the outer contour as the auxiliary. By changing the radius of the vessel to simulate changes in cerebral blood flow, potential changes were detected in the extracranial layer. The impedance and related cerebral blood flow parameters were calculated by the potential value, and the brain impedance measurement experiment was 
designed to verify the simulation results. The experimental results show that the impedance distribution of the brain in different blood supply states can be obtained, and there is a high consistency between the experimental results and the simulation values. The simulation model in this study can well analyze the autonomous regulation of cerebral blood flow and impedance variation, and lay a foundation for further analysis of cerebral blood flow problems.

\section{References}

1. B. Tyfa, D. Obidowski, P. Reorowicz. Biocybern Biomed Eng, 38 (02):228-242, (2018).

2. S. Acosta, D. J. Penny, K. M. Brady, C.G. Rusin. Microvasc Res, 115 (01): 34-43, (2018).

3. A. Metzger, J. Mulligan. Mil Med, 183 (03):119-123, (2018).

4. B.N. Cuffin, D. Cohen. BIOCYBERN Biocybern Biomed Eng, 47 (02):132-146, (1979).

5. L. Ke, L. Zhao, Q.Du. J Simul, 26 (03):517-522, (2014).

6. R. Cohen, S. Abboud. Med Eng Phys, 37 (05):453-459, (2015).

7. H. Christopher, S. Martin. Acta Neurochir, 126 (01):89-92, (2018).

8. Y. Zha, G.D Liu, Y.X Wang. Spectrosc Spect Anal, 37 (04):1069-1073, (2017).

9. R.S. Shah, D.S. Jeyaretna. Surgery, 36 (11 ):606-612, (2018).

10. J. Benathan-Tordjmann, P. Bailly. Comput Meth Prog Bio, 116 (03): 177-183, (2014).

[1] .
11. N. Nithya, L. Charles. Med Eng Phys, 31 (02):254-260, (2008).

12. B. Saugel, M. Cecconi, J.Y. Wagner, D.A. Reuter. Brit J Anaesth, 114 (04):22-25，(2015）.

13. G. Baltsavias, J. Richter. Word Neurosurg, 88 (04) :41-48, (2016).

14. O. Onaizah, T.L. Poepping, M. Zamir. Med Eng Phys, 49 (11) :121-130, (2017).

15. L. Ke, P.P Li, H. Chen. Chin J of Biomedical Eng, 34 (05): 566-573, (2015).

16. L. Wang, R.G Liu. Med health equipment, 34 (03) :4-6, (2013).

17. K. Yung-Bin, J. Dahee. J Therm Biol, 69 (07) :132-138, (2017).

18. L. Ke, Y.Z Gao. Chin J of Biomedical Eng, 35 (01):55-62, (2016).

19. Y.S Zeng, C.H. Xu. Chin Med equipment, 34 (01) :10-13, (2019).

20. D.D Yan, S.Y Shen. J of Chin Metrology Institute ,26 (03) :331-335, (2015).

21. H.M. Li, S.Y. Shen. J of Bio Eng, 33 ( 05 ):998-1004, (2016).

22. C.H Xu, T. Zhang. Chin Med equipment, 30( 07):5-7, (2015).

23. N.B. Vida, J. Siyavash. Life sciences, 209 (09) :202-209, (2018).

24. B. Saugel, M. Cecconi. Brit J Anaesth, 114 (04) :562-575, (2015)

25. N. Bowler, D. Shamley, R. Davies. Manual Ther, 16 (01):87-93, (2010) 